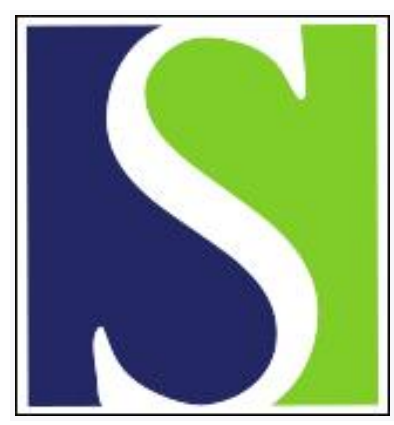

Scand J Work Environ Health 1994;20(1):62-64

https://doi.org/10.5271/sjweh.1427

Issue date: $01 \mathrm{Feb} 1994$

Is there an association between pleural plaques and lung cancer without asbestosis?

by Nurminen $\mathrm{M}$, Tossavainen $\mathrm{A}$

Affiliation: Department of Epidemiology and Biostatistics, Finnish Institute of Occupational Health, Helsinki.

This article in PubMed: www.ncbi.nlm.nih.gov/pubmed/8016601

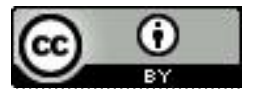




\title{
Is there an association between pleural plaques and lung cancer without asbestosis?
}

\author{
by Markku Nurminen, PhD, ${ }^{1}$ Antti Tossavainen, $\mathrm{DTech}^{2}$
}

\begin{abstract}
NURMINEN M, TOSSAVAINEN A. Is there an association between pleural plaques and lung cancer without asbestosis? Scand J Work Environ Health 1994;20:62-4. OBJEcTIVES - A recent review or meta-analysis of epidemiologic studies concluded that persons with asbestos-related pleural plaques do not have an increased risk of lung cancer in the absence of parenchymal asbestosis. The reviewer inferred that this conclusion provided indirect supportive evidence for the proposition that asbestosis is a necessary precursor of asbestos-related lung cancer. The objective of the present communication is to contest these claims. METHODs - Finnish epidemiologic data and population statistics were used to estimate the apparent risk ratio of lung cancer associated with radiographic signs of pleural plaques. Power calculations were applied to compute the needed population sizes to demonstrate that the association is statistically significant. RESULTS - Unrealistically large population studies would be needed to observe the statistical relation between pleural plaques and lung cancer, quantitated as a risk ratio of 1.1 , resulting from relatively low levels of environmental asbestos exposure. In realistic and valid epidemiologic studies on heavily exposed subpopulations, a two- or threefold risk can be identified. Conclusions - Uninformative studies should not be interpreted as providing suppressive evidence that pleural plaques are a noncausal risk indicator of lung cancer. Even for the null hypothesis, the inference that asbestosis is a necessary causal link between asbestos and lung cancer is illogical.
\end{abstract}

Key terms: epidemiologic studies, pulmonary tumors, risk assessment, statistical inference.

In a recent review, Weiss (1) examined epidemiologic studies that dealt with the relationship between pleural plaques unaccompanied by asbestosis and lung cancer. He used the method of detecting pleural disease to exclude asbestosis. In all, there were 13 investigations, six cohort studies, four lung cancer case-referent studies, and three autopsy studies. Weiss judged that only three studies supported the hypothesis that lung cancer risk is elevated among persons with pleural plaques over the risk of unexposed people and that these three studies had the most defects in design. Accordingly, Weiss concluded that "... persons with asbestos-related pleural plaques do not have an increased risk of lung cancer in the absence of parenchymal asbestosis [p 1854]." This conclusion is questionable. Weiss argued that the weight of evidence favors the hypothesis because the examined studies either yielded a "negative" result or were invalidated by a deficient design. This argument is specious. While Weiss acknowledged that it is difficult (in principle impossible) to prove a negative proposition in empirical studies, he neglected to consider the important issue of statistical power.

1 Department of Epidemiology and Biostatistics, Finnish Institute of Occupational Health, Helsinki, Finland.

2 Department of Industrial Hygiene and Toxicology, Finnish Institute of Occupational Health, Helsinki, Finland.

Reprint requests to: Dr M Nurminen, Department of Epidemiology and Biostatistics, Finnish Institute of Occupational Health, Topeliuksenkatu 41 a A, FIN-00250 Helsinki, Finland.

\section{A priori epidemiologic and medical knowledge}

Pleural plaques are undoubtedly related to asbestos exposure. Simultaneously, the incidence of lung cancer increases with asbestos exposure. The epidemiologic results indicate that there should exist an association (statistical correlation) between the occurrence of plaques and the realized risk of lung cancer. In other words, the presence of pleural plaques is a sign that conveys predictive information about the presence or future occurrence of lung cancer without a causal connotation (ie, a noncausal risk indicator of lung cancer). But it is established medical knowledge that pleural plaques are not a precursor of lung cancer. Most lung cancers are diagnosed without any sign of pleural plaques, and lung parenchyma and the parietal pleura are anatomically and histologically different. On the other hand, whether asbestosis is an intermediate stage in the causal process from asbestos exposure to the development of lung cancer is a matter of some contention in the literature.

\section{Weiss' claims}

Weiss (1) addressed the question of a possible mechanism of asbestos-induced lung cancer. He considered the following two possible models: one with asbestosis as an intermediate stage in the pathogenesis and the other with pleural plaques as a link in the causal pathway leading to lung cancer. To discriminate between the models, Weiss indirectly inferred that, if the risk of lung cancer is not increased for persons with asbestos-related pleural plaques but 
without asbestosis, then asbestosis would be a necessary precursor for asbestos-related lung cancer. In this argument, pleural plaques were used as a marker of asbestos exposure among asbestos-exposed persons. Even though the estimate of relative risk ${ }^{3}$ would not be statistically significantly elevated for such persons in comparison with the risk for unexposed persons or the general population, Weiss' hypothesis is not necessarily correct.

\section{Counterclaims to Weiss' claims}

We have three counterclaims to Weiss' claims. First, the validity (ie, sensitivity plus specificity) of pleural plaques as a marker of asbestos exposure is usually poor. The sensitivity of pleural plaques is often low. This insensitivity stems from the fact that not all persons with asbestos exposure develop pleural plaques that originate from the exposure, as is evident, for example, from the autopsy studies reviewed by Weiss. Regarding the specificity issue, not all pleural plaques are asbestos-related; instead, the radiological signs can be due to, for example, tuberculosis. Hypothetically, if pleural plaques were a perfectly valid marker of asbestos exposure, then all asbestos-exposed subjects would be marked as such, and no asbestos-related plaques would be detected in unexposed subjects. Weiss limited the selected studies to comprise asbestos-exposed subjects only, but he neglected to consider the validity of pleural plaques as a biological marker.

Second, from the rejection of a model with the pleural plaques link it does not follow that asbestosis is a necessary precursor among asbestos-exposed subjects. If asbestosis were a necessary condition, then every lung cancer case attributable to asbestos would also involve asbestosis. Unfortunately, one cannot identify the exposure-induced cases without resorting to strong biological assumptions, but one can estimate the proportionate increase in case load, the excess fraction, due to exposure (2). If the relative lung cancer risk, $R$, of an asbestos-exposed population is, for example, 2.0, then the excess cases attributable to exposure form the fraction (3), $(\mathrm{R}-1) \mathrm{R}^{-1}$, of all cases occurring in the exposed group, which is 0.5 ; thus, with biases (eg, confounding by smoking) absent, the proportion of asbestosis in the exposed population would be $50 \%$. But epidemiologic data contradict this estimate $(4,5)$. (See table 1.) Consequently, the claim does not hold.

In addition, when both asbestosis and lung cancer are found together, because of the long latency time of these diseases, it can be difficult to ascertain that asbestosis preceded lung cancer. Moreover, there are alternatives to the two-stage models. A simple model assumes that asbestos exposure is a common etiologic agent causing the pleural plaques on

Relative risk is used here as a generic term for risk ratio, rate ratio, or hazard ratio regardless of the study design which yielded the estimate. one hand, and the fibrogenic and oncogenic effects in human lung on the other hand, independently of each other without an intermediate stage. (For discussion, see reference 6 and the references therein.) Recent evidence suggests that lung tumors can be caused by relatively low levels of asbestos exposure independently of parenchymal fibrosis $(5,7)$.

Third, there remains the core question of how large a population is needed in a valid epidemiologic study to demonstrate the association between the occurrence of pleural plaques and an excess risk of lung cancer. We have attempted to answer this question of statistical power by considering an empirical example.

\section{Quantitation of lung cancer risk and power calculations}

In a representative sample of 3274 adult Finnish men, the proportions definitely or probably, possibly, and unlikely exposed to asbestos were estimated to be $13.4,52.9$, and $33.7 \%$, respectively (8). In these exposure categories, the corresponding prevalences of radiographic pleural plaques were $13.0,6.5$, and $4.6 \%$, respectively. In two other studies, the risk of dying from lung cancer among asbestos-exposed men was about twofold $(4,7)$. From these findings we computed the relative risk of lung cancer associated with the presence of pleural plaques to be as low as 1.1 (table 2). Upon adjustment by smoking cate-

Table 1. Cross-classification of lung cancer and asbestosis as the underlying or contributory causes of death on the death certificates of members of a cohort of male asbestos workers ${ }^{a}$ showing that lung cancer can appear without asbestosis and vice versa in asbestos-exposed persons. ${ }^{b}$

\begin{tabular}{|c|c|c|c|}
\hline & Asbestosis & No asbestosis & Total \\
\hline $\begin{array}{l}\text { Lung cancer } \\
\text { No lung cancer }\end{array}$ & $\begin{array}{r}3 \\
22\end{array}$ & $\begin{array}{r}10 \\
193\end{array}$ & $\begin{array}{r}13 \\
215\end{array}$ \\
\hline Total & 25 & 203 & 228 \\
\hline
\end{tabular}

a From reference 4 .

b Proportion of asbestosis is $25: 228=11 \%$.

Table 2. Cross-classification of a population sample of 3274 men according to the likelihood of asbestos exposure and the presence of pleural plaques. ${ }^{a}$ On the assumption that the risk of lung cancer is twofold among the probably exposed men relative to that among the possibly or unlikely exposed men, the apparent relative risk associated with pleural plaques can be computed simply as the ratio of prevalent numbers weighted by the specific risk of the asbestos exposure category. ${ }^{b}$

\begin{tabular}{lccr}
\hline Asbestos exposure & $\begin{array}{c}\text { With } \\
\text { plaques }\end{array}$ & $\begin{array}{l}\text { Without } \\
\text { plaques }\end{array}$ & Total \\
\hline Probably exposed & 57 & 382 & 439 \\
Possibly or unlikely & 164 & 2671 & 2835 \\
\hline Total & 221 & 3053 & 3274 \\
\hline
\end{tabular}

a From reference 6 .

b Relative risk $=[(57 \times 2+164 \times 1) / 221] /[(382 \times 2+2671 \times 1) /$ $3053]=1.1$. 
gories, the relative risk was exactly the same, 1.1. To show that such a relative risk is statistically significant, one would need to study a representative population sample of approximately 300000 people, which would render the study prohibitively expensive.

One of the studies reviewed by Weiss (1) was a tuberculosis screening survey conducted in three communities in Finland. It enrolled 7986 adults who were followed for 18 years (9). The communities represented different patterns of residential and occupational exposure to asbestos. By no means were all of the subjects with pleural plaques exposed to asbestos, as reported by Weiss. (See tables 1 through 3 in reference 1.) Moreover, not all of the pleural abnormalities in the asbestos-exposed persons were asbestos-related pleural plaques, as assumed by Weiss in his inference. The observed number of lung cancer cases among the 604 subjects with localized pleural plaques but no other asbestos-related radiographic signs was 28 , whereas, among the 604 reference persons with no signs of pleural abnormalities (some of whom could have been exposed to asbestos), 25 lung cancers were identified. The gender-, age-, and residence-adjusted relative risk was 1.1 - an estimate similar to the aforementioned one - with a $95 \%$ confidence interval of $0.6-1.8$. Information on smoking habits was not available. Because of the generally low levels of environmental asbestos exposure, it is unlikely that an expanded study base (ie, larger population size or longer follow-up time) that would be practical would demonstrate a statistically significantly elevated risk of lung cancer among the carriers of pleural plaques.

If, instead, the study had focused on a definitely or probably asbestos-exposed subpopulation, then a sample size calculation with the same statistics and estimates would yield the following result: with a significance level of $5 \%$ and a power of $80 \%$ the required sizes of the asbestos-exposed and an equalsized unexposed comparison group would have to be $538+538$ or $175+175$ to detect an apparent relative risk of 2 or 3, respectively. (See reference 10.) These sample sizes are tractable.

\section{Concluding remarks}

In closing, we caution against drawing hasty conclusions because some of the results of "negative" stud- ies on asbestos-exposed populations have turned out to be questionable or based on inadequate population-time experience. In realistic epidemiologic studies, selection bias, uncontrolled confounding, misclassification of exposure (and often disease), and sampling variability are especially difficult to quantify. Presumably, most of these uncertainties distort the pleural plaque-lung cancer association in the negative direction. (For a discussion, see reference 9.) Finally, before conclusions are drawn, the logic of inference from epidemiologic evidence to the probability of causation should be flawless.

\section{References}

1. Weiss W. Asbestos-related pleural plaques and lung cancer. Chest 1993;103:1854-9.

2. Robins JM, Greenland S. Estimability and estimation of excess and etiologic fractions. Stat Med 1989;8: $845-59$.

3. Greenland S, Robins JM. Conceptual problems in the definition and interpretation of attributable fractions. Am J Epidemiol 1988;128:1185-97.

4. Nurminen M. A study of the mortality of workers in an anthophyllite asbestos factory in Finland. Work Environ Health 1972;2:112-8.

5. Karjalainen A, Anttila S, Heikkilä L, Kyyrönen P, Vainio $H$. Lobe of origin of lung cancer among asbestos-exposed patients with or without diffuse interstitial fibrosis. Scand J Work Environ Health 1993;19: $102-7$.

6. Rubin AH. Common problems in asbestos-related pulmonary diseases. Am J Ind Med 1986;10:555-62.

7. Anttila S, Karjalainen A, Taikina-Aho $\mathrm{O}$, Kyyrönen $\mathrm{P}$, Vainio H. Lung cancer in the lower lobe is associated with pulmonary asbestos fiber count and fiber size. Environ Health Perspect 1993;101:166-70.

8. Tossavainen A, Karjalainen A, Impivaara O, Zitting A. Job title as a measure of occupational exposure to asbestos in an adult population sample. In: International Agency for Research on Cancer (IARC). Book of abstracts of the Conference on Retrospective Assessment of Occupational Exposures in Epidemiology. Lyon: IARC. In press.

9. Partanen T, Nurminen M, Zitting A, Wiikeri M, Ahlman K. Localized pleural plaques and lung cancer. Am J Ind Med 1992;22:185-92.

10. Rösler J-A, Woitowitz H-J, Lange H-J, Ulm K, Woitowitz RH, Rödelsperger K. Radiologic diagnosis of asbestosis and risk for asbestos-related malignancy. In: Proceedings of the eighth international conference on occupational lung diseases; vol 1, September 1992, Prague, Czechoslovakia. 1993:357-67.

Received for publication: 8 November 1993 